\title{
Escape paths of Besicovitch triangles
}

\author{
Yevgenya Movshovich and John E. Wetzel
}

\begin{abstract}
The shortest arcs that do not fit in the interior of a compact, convex body in the plane have been called its escape paths. We summarize some basic general facts about such paths. Specializing to triangles, we study the escape paths of the so-called Besicovitch triangles, near-equilateral isosceles triangles recently shown by Coulton and Movshovich to be covers for the family of all arcs of unit length, and we show that each such triangle has exactly one escape path.

AMS 2010 SUBJECT CLASSIFICATIOns: Primary 52C15; secondary 05B99. KEYWORDS AND PHRASES: Worm problem, escape path, Besicovitch triangle.
\end{abstract}

Shortest arcs that do not fit in the interior of a compact, convex body $\boldsymbol{K}$ in the plane are its "escape paths", in the language of the "lost in a forest" boundary search problems, and their common length $\varepsilon(\boldsymbol{K})$ is called its "escape length". If one thinks of $\boldsymbol{K}$ as a "forest" of known shape and size in which one is completely lost, an escape path is the shortest path one can follow to be assured of reaching the boundary $\partial \boldsymbol{K}$ of $\boldsymbol{K}$, no matter where one is in the forest or in which direction one sets out. The notion apparently is due to Bellman [1]. For a recent overview of such boundary search problems, see Finch and Wetzel [6].

Escape paths also play a role in arc-covering problems, the so-called "worm" problems, because one can show that if $\boldsymbol{K}$ has escape length $\varepsilon$, then $\boldsymbol{K}$ contains a congruent copy of every arc of length $\varepsilon$, i.e., $\boldsymbol{K}$ is a cover for the family $\mathcal{C}_{\varepsilon}$ of all such arcs. For a look at some arc-covering problems, see Wetzel [17] and [16].

Although elementary compactness considerations guarantee the existence of escape paths for any compact, convex body, the determination of their precise shape is generally not easy.

Graham [7] asked nearly 50 years ago for the shortest arc that does not fit in an open equilateral triangle of side one. Two years later, Besicovitch [2] conjectured that a certain $z$-shaped arc is as short as such an arc can be. The arc Besicovitch described is a centrally symmetric polygonal arc formed by three line segments each of length $\sqrt{3 / 28}$. 
Restated as a covering problem, the Besicovitch conjecture is that an equilateral triangle of side $\sqrt{28 / 27} \approx 1.018$ is a cover for the family $\mathcal{C}_{1}$ of all unit arcs in the plane, i.e., it contains a congruent copy of every planar arc of length 1 . This conjecture was recently proved by Coulton and Movshovich [3], who studied more generally a certain family of near-equilateral isosceles triangular regions and determined for each the smallest dimensions large enough to cover $\mathcal{C}_{1}$. They called those smallest triangles Besicovitch triangles.

In this paper, after discussing some basic properties of escape paths in general, we investigate the escape paths of Besicovitch triangles and prove that up to congruence, each such triangle has a unique escape path.

\section{Escape paths}

We begin by establishing our notation and some basic facts about escape paths. An oval $\boldsymbol{K}$ is a compact, convex set with non-empty interior. We write $\partial \boldsymbol{K}$ for its (topological) boundary, $\partial \boldsymbol{K}=\boldsymbol{K} \backslash \operatorname{int} \boldsymbol{K}$. We assume paths ${ }^{1}$ to be rectifiable and parameterized on arclength, and we write $l(\gamma)$ for the length of the path $\gamma$. We do not bother to make a notational distinction between path as function and path as range of the function.

Definition. A path $\gamma$ is an exit path for an oval $\boldsymbol{K}$ if for each point $I$ in int $\boldsymbol{K}$ and each Euclidean motion $\mu$ for which $\mu(\gamma(0))=I$,

$$
\{\mu(\gamma(s)): 0 \leq s \leq l(\gamma)\} \cap \partial \boldsymbol{K} \neq \emptyset .
$$

In less formal terms, a path is an exit path of an oval $\boldsymbol{K}$ if no matter how it is placed with its initial point inside $\boldsymbol{K}$, it invariably meets the boundary of $\boldsymbol{K}$.

It is not difficult to show that if $\gamma$ is an exit path for $\boldsymbol{K}$, if the point $I$ lies on the boundary $\partial \boldsymbol{K}$ of $\boldsymbol{K}$, and if $\mu$ is a motion so that $\mu \circ \gamma(0)=I$ and the transplanted path $\mu \circ \gamma$ meets $\operatorname{int} \boldsymbol{K}$, then $\{\mu(\gamma(s)): 0<s \leq l(\gamma)\} \cap \partial \boldsymbol{K} \neq \emptyset$. So any path that does not fit in the oval is an exit path for the oval. An oval always has exit paths - for example, any line segment whose length is at least the diameter of $\boldsymbol{K}$ is an exit path.

Definition. The escape length $\varepsilon(\boldsymbol{K})$ of an oval $\boldsymbol{K}$ is the greatest lower bound of the lengths of its exit paths. An escape path of $\boldsymbol{K}$ is an exit path of length $\varepsilon(\boldsymbol{K})$.

\footnotetext{
${ }^{1}$ We regard the terms arc, curve, and path as synonyms.

${ }^{2}$ Our terminology here differs somewhat from that employed in Finch and Wetzel $[6]$.
} 
A path is an exit path for an oval $\boldsymbol{K}$ if and only if it does not fit in the interior of $\boldsymbol{K}$ (an assertion that could be taken as the definition). Hence, as described in the first paragraph of the article, every shortest path that does not fit in the interior of an oval $\boldsymbol{K}$ is an escape path of $\boldsymbol{K}$.

The escape length of an oval is a fundamental geometric measure associated with the oval that, like area, perimeter, minimum width, and diameter, is monotonic with respect to set inclusion. Its ultimate importance remains unclear. The following three basic theorems are proved in Finch and Wetzel [6].

Theorem 1. Let $\boldsymbol{K}$ be an oval and $\gamma$ a path in $\boldsymbol{K}$. If (a) $\gamma$ does not fit in $\operatorname{int} \boldsymbol{K}$, but (b) every path strictly shorter than $\gamma$ fits in $\operatorname{int} \boldsymbol{K}$, then $\gamma$ is an escape path for $\boldsymbol{K}$.

Theorem 2. If every path of length less than $L$ fits in $\boldsymbol{K}$, then every path of length $L$ fits in $\boldsymbol{K}$.

Theorem 3. The escape length $\varepsilon$ of $\boldsymbol{K}$ is the largest $L$ for which $\boldsymbol{K}$ is a cover for the family $\mathcal{C}_{L}$ of all arcs in the plane of length at most $L$. In particular, $\boldsymbol{K}$ is a cover for $\mathcal{C}_{\varepsilon(\boldsymbol{K})}$.

It frequently is convenient to scale the oval to have escape length one, so that the scaled oval is a cover for the family $\mathcal{C}_{1}$ of all arcs of unit length.

Norwood, Poole, and Laidacker [13], refining an earlier cover for the family $\mathcal{C}_{1}$ of all unit arcs found by Poole and Gerriets [14] in 1973, showed in 1992 that the region pictured in Figure 1 is a cover for $\mathcal{C}_{1}$. This region is formed by clipping an end of the minor diagonal of a $60^{\circ}$ rhombus of side $1 / \sqrt{3}$ with a circular arc of radius $\frac{1}{2}$ (see Wetzel [17]). Since this cover has diameter 1 , it has escape length $\varepsilon=1$. This oval has uncountably many non-congruent escape paths, four of which are drawn in Figure 1.

Not much is known about the escape paths of ovals in general:

1. An escape path need not be polygonal, even if the oval has a polygonal boundary (see [6, p. 649]).

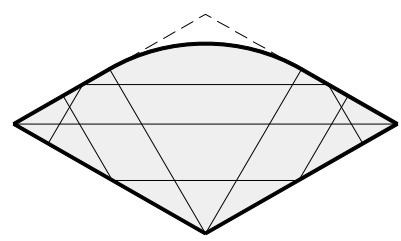

Figure 1: The NPL cover. 
2. There is an oval that has exactly two non-congruent escape paths (see [6, p. 649]). It is not known whether an oval can have exactly $n$ noncongruent escape paths for any $n>2$.

3. An escape path need not be convex (see, for example, the Besicovitch $\mathfrak{z} \alpha$, below).

4. It seems likely that an escape path must be a simple arc with distinct endpoints.

\section{The case of a triangle}

If the oval is a triangle (that is to say, a closed triangular region), escape paths can be characterized in the following convenient way. We agree that line segments always include their endpoints.

Definition. (cf. [3, p. 82]) A path $\gamma$ in a triangle $T$ is an extreme path of $T$ if it and every path in $T$ congruent to it meet each of the three sides of $T$.

Lemma 4. If a triangle $T$ has escape length $\varepsilon(T)$, then $l(\gamma) \geq \varepsilon(T)$ for every extreme path $\gamma$ in $T$; and an extreme path of length $\varepsilon(T)$ is an escape path.

Proof. If an escape path $\gamma$ in $T$ does not meet one side of $T$, then a small translation toward that side moves it into the interior of $T$, contrary to the definition of exit path. Hence every escape path in $T$ must be an extreme path of $T$, and $l(\gamma) \geq \varepsilon(T)$. On the other hand, suppose a path $\gamma$ of length $\varepsilon(T)$ in $T$ is extreme, let $I$ be an interior point of $T$, let $\mu$ be a motion that carries $\gamma$ so that its initial point is $I$, and let $\gamma_{0}=\mu \circ \gamma$. If $\gamma_{0}$ fits in $T$, then it meets $\partial T$ by hypothesis, and if $\gamma_{0}$ does not fit in $T$, then it surely meets $\partial T$. Hence $\gamma$ is an exit path of length $\varepsilon(T)$ and consequently an escape path.

In other words, every extreme path $\gamma$ of a triangle $T$ has length at least $\varepsilon(T)$, and an extreme path has length $\varepsilon(T)$ only if it is an escape path.

\subsection{The triangle $T_{\alpha}$ and the path $\mathfrak{z}_{\alpha}$}

Recall the classical language for an isosceles triangle $T$ : if $T$ is not equilateral, the two equal sides are the "arms" of the triangle and the third side is the "base"; but if $T$ is equilateral, each side is both an "arm" and a "base" of $T$. Each side of $T$ includes its endpoints. In the remainder of this article we denote by $T_{\alpha}$ the isosceles triangle $X Y Z$ with base $X Y$ and base angle $\alpha$ whose base has length

$$
b_{\alpha}=X Y=\frac{1}{3} \sqrt{9+\cot ^{2} \alpha}
$$




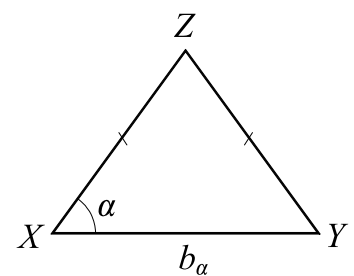

(a) Triangle $T_{\alpha}$.

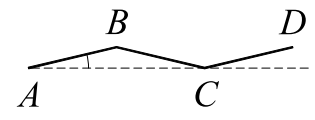

(b) $\mathfrak{z} \alpha$.

Figure 2

and whose vertices are labeled in the counterclockwise sense (Figure 2(a)).

For given $\alpha$ a certain three-segment polygonal arc plays an important role.

Definition. The Besicovitch "zee" $\operatorname{arc} \mathfrak{z} \alpha$ is the centrally symmetric threesegment polygonal unit arc $\langle A B C D\rangle$, with $A B=B C=C D=\frac{1}{3}, A B \|$ $C D$, and $\angle C A B=\arctan \left(\frac{1}{3} \cot \alpha\right)$ (Figure $\left.2(\mathrm{~b})\right)$.

One can easily verify that for $\mathfrak{z} \alpha$,

$$
\begin{aligned}
A C & =\frac{2 \tan \alpha}{\sqrt{1+9 \tan ^{2} \alpha}}, \\
A D & =\frac{1}{3} \sqrt{\frac{1+81 \tan ^{2} \alpha}{1+9 \tan ^{2} \alpha},} \\
\angle C A D & =\arctan \frac{1}{9} \cot \alpha .
\end{aligned}
$$

Definition. A triangle $T_{\alpha}$ is a Besicovitch triangle ${ }^{3}$ if it covers every unit arc and if it is the smallest such triangle in its similarity class.

The main result of Coulton and Movshovich [3] is that for $\alpha$ in the range

$$
52.239^{\circ} \approx \arctan \sqrt{\frac{5}{3}} \leq \alpha \leq \arctan \sqrt{3}=60^{\circ},
$$

$T_{\alpha}$ is a cover for the family $\mathcal{C}_{1}$ of all unit arcs in the plane:

Theorem 5. [3] Triangle $T_{\alpha}$ is a Besicovitch triangle for $\alpha$ in the range (3).

\footnotetext{
${ }^{3}$ This definition is a little different from that employed in [3].
} 


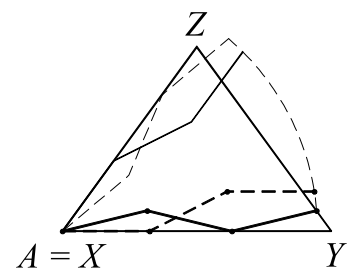

(a) Vertex fit.

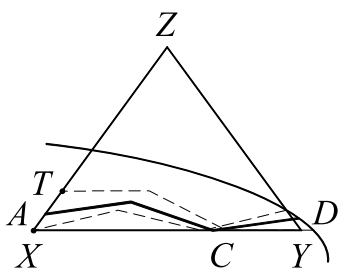

(b) No other fit.

Figure 3: The arc $z_{\alpha}$ in $T_{\alpha}$.

Proof. The authors of [3] showed directly that $T_{\alpha}$ contains a congruent copy of every simple polygonal unit arc, and they concluded from an approximation covering theorem in $\left[9\right.$, Theorem 14] that $T_{\alpha}$ is a cover for all unit arcs. That it is the smallest such triangle in its similarity class follows because $\mathfrak{z} \alpha$ fits in $T_{\alpha}$ only as pictured in Figure 3(a), with one endpoint at a base vertex of $T_{\alpha}$ and the third vertex on the base of $T_{\alpha}$.

It is conceivable that $\mathfrak{z}_{\alpha}$ might also fit in $T_{\alpha}$ as pictured in Figure $3(\mathrm{~b})$, with $A$ near $X$ on $X Z, C$ on $X Y$, and $D$ inside $T_{\alpha}$ near $Y$, a case not considered in [3]. We take this opportunity to show that this cannot happen, thereby correcting a small lacuna in the argument in [3].

Recall the following classical locus, which Dörrie [4, pp. 214-216] attributes to the Dutch mathematician Frans van Schooten the younger (16151660). (See, for example, Honsberger [8, pp. 173-177] or Wetzel [15].)

Schooten's Locus. If the vertices $P$ and $Q$ of a triangular tile $P Q R$ move respectively on two intersecting lines $p$ and $q$, the locus of the third vertex $R$ is a (possibly degenerate) ellipse whose center is at the point in which the lines $p$ and $q$ meet.

If $A=X$, a short calculation shows that the endpoint $D$ lies in $T_{\alpha}$ only when $C$ lies on the base $X Y$ (as pictured in Figure 3(a)); and similarly if $A=Y$. If $A$ is neither $X$ not $Y$, then by a suitable motion we may suppose that $A$ lies on $X Z$ and $C$ lies on the base $X Y$ (Figure 3(b)). We argue that then $D$ must extend beyond $Y Z$. Indeed, the locus of the point $D$ as $A$ moves on the line $X Z$ and $C$ moves on the line $X Y$ is an ellipse centered at $X$ (Figure 3(b)). Let $T$ be the point on $X Z$ for which $D$ lies on the baseline $X Y$. Then the elliptic arc traced when $A$ moves from $X$ to $T$ is convex and has its initial point on $Y Z$ and its terminal point on the line $X Y$ outside the triangle. It follows that apart from its initial point on $Y Z$ it lies entirely across $Y Z$ from $X$. 
This completes the proof that the Besicovitch $\operatorname{arc} \mathfrak{z}_{\alpha}$ fits in $T_{\alpha}$ only along a base, and it follows that $T_{\alpha}$ is a Besicovitch triangle when its base angle $\alpha$ falls in the range (3).

Corollary 6. The Besicovitch arc $\mathfrak{z}_{\alpha}$ is an escape path of the oval $T_{\alpha}$.

Proof. The proof shows that $\mathfrak{z} \alpha$ is an extreme path of length 1 , and the result follows from Lemma 4.

We write

$$
\alpha_{0}=\arctan \sqrt{\frac{5}{3}} \approx 52.24^{\circ},
$$

so that

$$
\arctan \left(\frac{1}{3} \cot \alpha_{0}\right)=\arctan \sqrt{\frac{1}{15}} \approx 14.48^{\circ} .
$$

Because the base $b_{\alpha}=\frac{1}{3} \sqrt{9+\cot ^{2} \alpha}$ of $T_{\alpha}$ is a decreasing function of $\alpha$ on the range (3),

$$
1.018350 \approx b_{60^{\circ}} \leq b_{\alpha} \leq b_{\alpha_{0}} \approx 1.032796 .
$$

We have chosen $\alpha=54^{\circ}$ for most of the drawings of $T_{\alpha}$ in this article.

\section{Various preliminaries}

Recall that a path $\gamma$ from $P$ to $Q$ is parameterized by arclength, so that $\gamma(0)=P$ and $\gamma(l(\gamma))=Q$. The path $\gamma$ from $P$ to $Q$ is open if $Q \neq P$. We begin by showing that an escape path must be open.

Lemma 7. An escape path of $T_{\alpha}$ has endpoints at least 0.59 apart.

Proof. A well-known theorem of Eggleston [5, p. 157] implies that every closed curve whose length is at most the circumference of the incircle of a triangle can be covered by that triangle. The circumference

$$
c(\alpha)=\frac{\pi \sqrt{1+9 \tan ^{2} \alpha}}{3(1+\sec \alpha)}
$$

of the incircle of the Besicovitch triangle $T_{\alpha}$ is strictly increasing on the range (3), so the smallest value it can take occurs at $\alpha_{0}=\arctan (\sqrt{5 / 3})$ :

$$
c(\alpha) \geq c\left(\alpha_{0}\right)=\frac{\pi \sqrt{1+9 \tan ^{2} \alpha_{0}}}{3\left(1+\sec \alpha_{0}\right)}>1.59 .
$$


If $\gamma$ is an escape path from $T_{\alpha}$ whose endpoints are $d<0.59$ apart, joining those endpoints with a line segment gives a closed path whose length is less than $c(\alpha)$, which consequently fits in the interior of $T_{\alpha}$, a contradiction that proves the result.

\subsection{The $\Lambda$-property}

It will be convenient to write $\operatorname{dist}(A, t)$ for the normal distance from a point $A$ to a line $t$.

Definition. An open arc $\gamma$ from $P=\gamma(0)$ to $Q=\gamma(l(\gamma))$ has the $\Lambda$-property if there are parameter values $b, c$, and $d$ with $0 \leq b<c<d \leq l(\gamma)$ and parallel lines $m$ and $n$ so that, writing $B=\gamma(b), C=\gamma(c)$, and $D=\gamma(d)$, (1) $B$ and $D$ lie on $m$, (2) $C$ lies on $n$, and (3) the arc $\gamma$ lies in the closed infinite strip $\sigma(m, n)$ between the lines $m$ and $n$ (Figure 4).

It was shown in [3] that every simple, open, polygonal arc has the $\Lambda$ property. Routine limiting arguments extend this result from simple polygonal arcs to arbitrary open arcs:

Theorem 8. Let $\gamma$ be an open arc of length L, parameterized on arclength. There are both a direction $\vartheta_{0}$ and a triple $(b, c, d)$ of real numbers $b, c$, and $d$ with $0 \leq b<c<d \leq L$ so that the points $B=\gamma(b)$ and $D=\gamma(d)$ lie on one of the two support lines in the direction $\vartheta_{0}$ and the point $C=\gamma(c)$ lies on the other (Figure 4). In other words, every open arc has the $\Lambda$-property. ${ }^{4}$

Proof. The claims are trivially correct if $\gamma$ is a subset of a line, so we suppose that the minimal width of $\gamma$ is positive. According to the simple approximation theorem [9, Th. 14] there is a sequence $\left\langle\varpi_{n}\right\rangle_{n=1}^{\infty}$ of simple polygonal arcs from $P$ to $Q$ such that for each $n=1,2, \ldots$,

$$
\begin{aligned}
\mathfrak{h}\left(\varpi_{n}, \gamma\right) & <\frac{1}{n}, \\
L-\frac{1}{n} & <l\left(\varpi_{n}\right) \leq L,
\end{aligned}
$$

where $\mathfrak{h}\left(\varpi_{n}, \gamma\right)$ is the Hausdorff distance between the (traces of ) $\varpi_{n}$ and $\gamma$. According to the proof of Theorem 5.1 of [3, pp. 86-87] there are sequences of angles $\vartheta_{n}$ (say in $[0,2 \pi]$ ) and parameter values $b_{n}, c_{n}$, and $d_{n}$ with $0 \leq$ $b_{n}<c_{n}<d_{n} \leq L$ so that the points $B_{n}=\gamma\left(b_{n}\right)$ and $D_{n}=\gamma\left(d_{n}\right)$ lie on one of the two support lines of $\varpi_{n}$ and the point $C_{n}=\gamma\left(c_{n}\right)$ lies on the other. There is a subsequence of angles that converges to an angle $\vartheta_{0}$ in $[0,2 \pi]$, call

\footnotetext{
${ }^{4}$ This is most likely a known result, but we have not succeeded in locating it in the literature.
} 


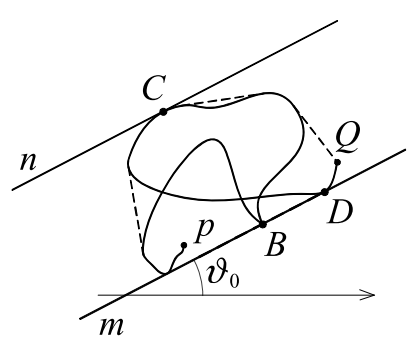

Figure 4: $\Lambda$ property.

it again $\left\langle\vartheta_{n}\right\rangle$; and by passing to another subsequence if necessary we may suppose that the support lines $m_{n}$ at angle $\vartheta_{n}$ converge to a support line $m$ at angle $\vartheta_{0}$ and the parallel support lines $m_{n}^{\prime}$ converge to the support line $m^{\prime}$ parallel to $m$ (say in the topology on lines induced from $\mathbb{R}^{3}$ by the association $p x+q y+r=0 \longleftrightarrow(p, q, r))$. Now for each positive integer $n, B_{n}$ and $D_{n}$ lie on $m_{n}$ and $C_{n}$ lies on $m_{n}^{\prime}$, or vice versa; and by passing to a subsequence if necessary we may suppose that $B_{n}$ and $D_{n}$ lie on $m_{n}$ and $C_{n}$ lies on $m_{n}^{\prime}$. Similarly we may arrange successively for $b_{n} \rightarrow b, c_{n} \rightarrow c$, and $d_{n} \rightarrow d$, where $0 \leq b \leq c \leq d \leq L$. Then $B_{n}=\varpi_{n}\left(b_{n}\right) \rightarrow B \in m \cap \gamma$, $D_{n}=\varpi_{n}\left(d_{n}\right) \rightarrow D \in m \cap \gamma$, and $C_{n}=\varpi_{n}\left(c_{n}\right) \rightarrow C \in m^{\prime} \cap \gamma$. Finally, because $\gamma$ has positive minimal width, neither of the equalities $b=c$ and $c=d$ can hold; and this completes the argument.

\subsection{Tetral arcs}

In this section we define a family of at-most four segment polygonal $\Lambda$-arcs in $T_{\alpha}$ that we need for comparison purposes in the final argument.

Let $a$ and $e$ be distinct points on the base $X Y$ of $T_{\alpha}$, let $b$ be a point on the $\operatorname{arm} X Z$ of $T_{\alpha}$ and $d$ a point on the arm $Y Z$, and let $c$ be a point in $T_{\alpha}$ but not on its base. We permit $b=a=X, d=e=Y$, and $c=b$ or $c=d$. It will be convenient to use the following notation: For points $x, y, z$ on an arc $\gamma$ we write $x \sim y \sim z$ to indicate that $y$ lies strictly between $x$ and $z$ in the arclength parametrization of $\gamma$, and $x \simeq y \sim z$ to indicate that either $y=x$ or $x \sim y \sim z$.

Definition. A tetral arc is an at-most four segment polygonal path in $T_{\alpha}$ with vertex set $\{a, b, c, d, e\}$ such that $a \sim c \sim e$ and $\operatorname{dist}(b, X Y) \leq$ $\operatorname{dist}(c, X Y)$ and $\operatorname{dist}(d, X Y) \leq \operatorname{dist}(c, X Y)$.

Note that every tetral arc has the $\Lambda$-property. 
3.2.1. Important families of tetral arcs Mirroring the development in [3], we study three important families of tetral arcs: staples, s-arcs, and $w$-arcs. We do not require that tetral arcs be simple.

Staples The staples are the least complicated of the tetral arcs.

Definition. A tetral arc is a staple if its vertices are in the order $a \simeq b \simeq$ $d \simeq e$, with $c=b$ or $c=d$.

Lemma 9. Every staple is longer than 1.

Proof. Suppose $\pi=\langle a b d e\rangle$ is a staple. Let $b^{\prime}$ and $d^{\prime}$ be the feet of the perpendiculars from $b$ and $d$ to the base $X Y$ of $T_{\alpha}$. Then

$$
l(\pi)=l(\langle a b d e\rangle) \geq l\left(\left\langle b^{\prime} b d d^{\prime}\right\rangle\right) \geq X Y>1
$$

because $\alpha \geq \alpha_{0}>45^{\circ}$.

Consequently staple arcs are too long to be escape paths.

$s$-arcs

Definition. A tetral arc is an $s$-arc if its vertices are in the order $a \simeq b \simeq$ $c \sim e \sim d$, with $d \neq Y$ (or the reverse: $b \simeq a \simeq c \sim e \simeq d$ and $b \neq X$ ).

Lemma 10. Every s-arc has length at least one. The Besicovitch arc $\mathfrak{z} \alpha$ is an $s$-arc of length one, and when $\alpha>\alpha_{0}$ it is the only $s$-arc of unit length. When $\alpha=\alpha_{0}$, there is an additional infinite family of s-arcs of unit length. The Besicovitch arc $\mathfrak{z}_{\alpha}$ is the only $s$-arc that is an escape arc for $T_{\alpha}$.

Proof. [3] Let $\rho_{1}$ and $\rho_{2}$ be the rays

$$
\begin{aligned}
& \rho_{1}: \quad y=3\left(b_{\alpha}-x\right) \tan \alpha, y \geq 0, \\
& \rho_{2}: \quad y=x \tan 2 \alpha, y \geq 0,
\end{aligned}
$$

in the upper half-plane. When $\alpha_{0}<\alpha \leq 60^{\circ}$, these rays are divergent, and their minimum distance is the length of the perpendicular from the origin to $\rho_{1}$, viz., 1 . When $\alpha=\alpha_{0}$ the rays $\rho_{1}$ and $\rho_{2}$ are parallel and the distance 1 apart.

Let $\pi$ be an $s$-arc having vertices $a, b, c, d, e$ in the order $a \simeq b \simeq c \sim$ $e \sim d$; the arguments in the reverse case are similar.

Suppose that $X, a, e, Y$ lie in that order on the base $X Y$ (Figure 5); the argument works as well if the points on the base are in the order $X, e$, $a, Y$. Let $a^{\prime}$ be the point on $\rho_{2}$ symmetric to $a$ in the $\operatorname{arm} X Z$. Let $h$ be 


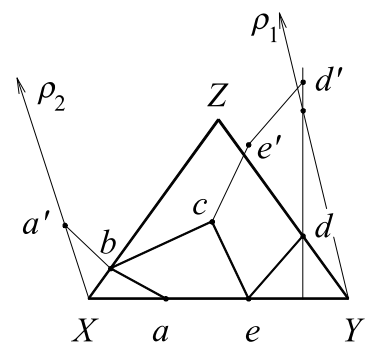

Figure 5: $X \sim a \sim e \sim Y$.

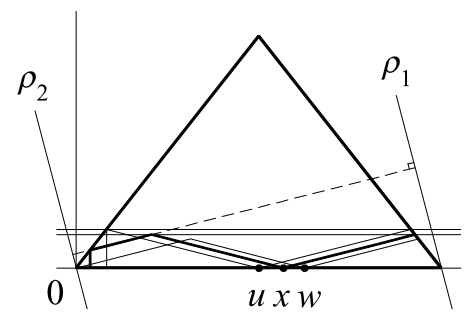

(a) The $s$-arcs.

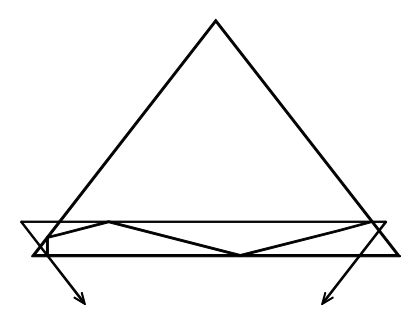

(b) The interior fit.

Figure 6: Unit $s$-arcs.

the ordinate of $c$, and let $e^{\prime}$ be the point above $e$ with ordinate $2 h$. Suppose $d=(x, y)$, and let $d^{\prime}$ be the point with coordinates $(x, y+2 h)$, so that the figure $d d^{\prime} e^{\prime} e$ is a parallelogram. Since $y \leq h$, the point $d^{\prime}$ lies on or above the ray $\rho_{1}$, and it follows that

$$
l(\pi)=a b+b c+c e+e d=a^{\prime} b+b c+c e^{\prime}+e^{\prime} d^{\prime} \geq_{1} a^{\prime} d^{\prime} \geq_{2} 1 .
$$

For the equality to hold in the first inequality $\left(\geq_{1}\right)$, the points $a^{\prime}, b, c, e^{\prime}$, $d^{\prime}$ must fall in that order on the line segment $a^{\prime} d^{\prime}$. If $\alpha>\alpha_{0}$ and the equality also holds in the second inequality $\left(\geq_{2}\right)$, then $a^{\prime} d^{\prime}$ must be the (unique) unit perpendicular from $\rho_{2}$ to $\rho_{1}$, which meets $\rho_{2}$ at $X$. Consequently $a^{\prime}=$ $a=X=b$, and it follows that $\pi=\mathfrak{z}_{\alpha}$. The reverse case is the symmetric argument.

When $\alpha=\alpha_{0}$ the rays $\rho_{2}$ and $\rho_{1}$ are 1 apart, and the equalities (4) require that $a^{\prime}$ and $d^{\prime}$ be the ends of a common perpendicular on $\rho_{2}$ and $\rho_{1}$ on which the points $a^{\prime}, b, c, e^{\prime}, d^{\prime}$ fall in that order. It follows that $l(\pi)=1$ is satisfied by an infinite family of $s$-arcs that includes $\mathfrak{z} \alpha$, pictured in Figure 6(a) and constructed by folding the unit segment (dashed in Figure 6(a) 


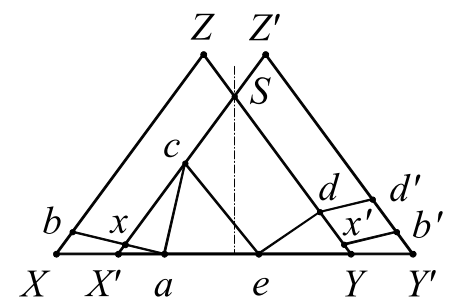

Figure 7: $\omega$-arcs.

by reflections. More precisely, let $\beta_{0}=\arctan \left(\frac{1}{3} \cot \alpha_{0}\right)$. For each $x$ between $u=\frac{1}{2} b_{\alpha_{0}} \approx 0.5164$ and $w=\frac{2}{3} \cos \beta_{0} \approx 0.6455$, position a flat " $\vee$ " opening upwards with vertex at $x$ on the base $X Y$, extend the right wing of the $\vee$ until it reaches the arm $Y Z$ and extend the left wing until its tip has the same height above the base as the tip of the right wing $t$. Then follow the perpendicular $t$ from $\rho_{1}$ to the left arm $X Z$ of $T_{\alpha_{0}}$ and thence down a perpendicular to the base $X Y$ (Figure 6(a), in which the extreme cases $x=u$ and $x=w$ are also drawn). Each such path has unit length, and when $x=w$ it coincides with $\mathfrak{z} \alpha$. In every other case the path fits in the interior of $T_{\alpha}$ inverted, as is clear in Figure 6(b). The reverse case adds nothing new.

It follows as claimed that the only $s$-arc that is an escape path is $\mathfrak{z} \alpha$.

$\boldsymbol{w}$ - and $\boldsymbol{w}^{*}$-arcs

Definition. A tetral arc whose vertices in the order $b \sim a \sim c \sim e \sim d$ is a $w$-arc when $\operatorname{dist}(b, X Y)+\operatorname{dist}(d, X Y) \leq \operatorname{dist}(c, X Y)$ and a $w^{*}$-arc when $\operatorname{dist}(b, X Y)+\operatorname{dist}(d, X Y)>\operatorname{dist}(c, X Y)$.

We will show that none of these arcs is an escape arc. We begin with $w$-arcs.

Lemma 11. Every $w$-arc has length at least 1. Every $w$-arc for which $\operatorname{dist}(b, X Y)+\operatorname{dist}(d, X Y)<\operatorname{dist}(c, X Y)$ is strictly longer than 1.

Proof. Let $\pi=\langle$ baced $\rangle$ be a $w$-arc. We place a copy $T_{\alpha}^{\prime}=X^{\prime} Y^{\prime} Z^{\prime}$ of $T_{\alpha}$ so that its arm $X^{\prime} Z^{\prime}$ just touches the segment $a c$ of $\pi$ (Figure 7). Suppose the segment $b a$ of $\pi$ meets the arm $X^{\prime} Z^{\prime}$ at $x$, and let $x^{\prime} b^{\prime}$ be the reflected image of $x b$ in the vertical line through $S$, the point $Y Z \cap X^{\prime} Z^{\prime}$. Let $d^{\prime}$ be the point so that the figure $d x^{\prime} b^{\prime} d^{\prime}$ is a parallelogram. Then $d^{\prime}$ lies on $Y^{\prime} Z^{\prime}$ and the tetral arc $\left\langle a c e d^{\prime}\right\rangle$ is an $s$-arc in $T_{\alpha}^{\prime}$ because

$$
\begin{aligned}
\operatorname{dist}\left(d^{\prime}, X^{\prime} Y^{\prime}\right) & =\operatorname{dist}\left(d, X^{\prime} Y^{\prime}\right)+\operatorname{dist}\left(b^{\prime}, X^{\prime} Y^{\prime}\right)-\operatorname{dist}\left(x^{\prime}, X^{\prime} Y^{\prime}\right) \\
& \leq \operatorname{dist}(d, X Y)+\operatorname{dist}(b, X Y) \leq \operatorname{dist}(c, X Y)
\end{aligned}
$$




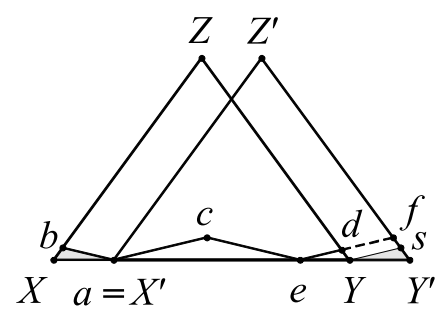

Figure 8: $\mathfrak{z} \alpha$-convolute.

Then according to Lemma 10,

$$
l(\pi)=l(\langle\text { baced }\rangle)=l\left\langle\text { xacedd } d^{\prime}\right\rangle \geq l\left(\left\langle\text { aced }^{\prime}\right\rangle\right) \geq 1 .
$$

Thus when $\operatorname{dist}(b, X Y)+\operatorname{dist}(d, X Y) \leq \operatorname{dist}(c, X Y)$, the $w$-arc $\pi$ has length at least 1; and when $\operatorname{dist}(b, X Y)+\operatorname{dist}(d, X Y)<\operatorname{dist}(c, X Y)$, then $\operatorname{dist}\left(d^{\prime}, X Y\right) \leq \operatorname{dist}(c, X Y)$, and the $w$-arc $\pi$ is longer than the $s$-arc $\left\langle a c e d^{\prime}\right\rangle$, which is longer than 1.

If $\pi$ is a $w$-arc of length 1 , Lemma 11 implies that $\operatorname{dist}(b, X Y)+\operatorname{dist}(d$, $X Y)=\operatorname{dist}(c, X Y)$, and it follows from (5) that the $s$-arc $\langle$ aced $\rangle$ has length 1. All such $s$-arcs are described in Lemma 10, and of those, only $\mathfrak{z} \alpha$ fits the geometric requirements of the present situation. When $\left\langle\right.$ aced $\left.^{\prime}\right\rangle=\mathfrak{z} \alpha$, it follows (Figure 7) that $a=X^{\prime}=x, a c=c e=e d^{\prime}=\frac{1}{3}$, and the generating angles ( $\angle e a c, \angle a e c$, and $\left.\angle Y^{\prime} e d^{\prime}\right)$ all equal the angle $\angle C A B$ of $\mathfrak{z}_{\alpha}$.

Definition. A $\mathfrak{z} \alpha$-convolute is a $w$-arc for which $\left\langle a c e d^{\prime}\right\rangle=\mathfrak{z} \alpha$.

Such arcs are easily constructed. Take any point $a$ on $X Y$ whose distance from $X$ lies between 0 and

$$
b_{\alpha}-\frac{2}{3 b_{\alpha}}=\frac{1}{3} \frac{\cot ^{2} \alpha+3}{\sqrt{\cot ^{2} \alpha+9}},
$$

and position $T_{\alpha}^{\prime}$ so that $X^{\prime}=a$ (Figure 8). Select points $c, e$, and $f$ so that $\langle$ ace $f\rangle=\mathfrak{z}_{\alpha}$, let $s$ be the point on $Y^{\prime} Z^{\prime}$ so that $Y s f d$ is a parallelogram, and take $b$ on $X Z$ so that $X b=Y^{\prime} s$. Then $b a=d f$, and the $w$-arc $\langle$ baced $\rangle$, which has length one, is a $\mathfrak{z} \alpha$-convolute of $\mathfrak{z} \alpha$.

None of these convolutes of $\mathfrak{z} \alpha$, however, is an escape path, because each fits in the interior of $T_{\alpha}$ :

Corollary 12. No $\mathfrak{z}_{\alpha}$-convolute other than $\mathfrak{z} \alpha$ itself is an escape path for $T_{\alpha}$. 


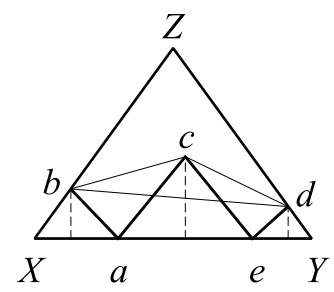

Figure 9: A $\mathfrak{z} \alpha$-convolute $\pi$.

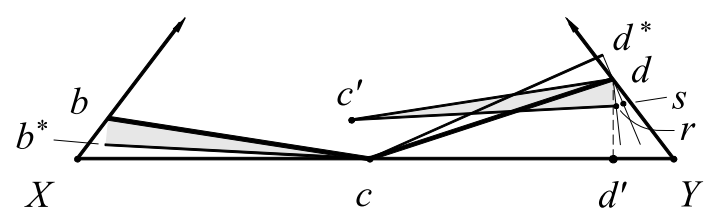

Figure 10: Proof of Corollary 12.

Proof. We show that the $\mathfrak{z}_{\alpha}$-convolute $\pi_{a}=\langle$ baced $\rangle$, inverted, fits in the interior of $T_{\alpha}$. To illustrate the argument we employ the vertically-exaggerated $w$-arc $\pi$ in Figure 9. The condition that

$$
\operatorname{dist}(b, X Y)+\operatorname{dist}(d, X Y)=\operatorname{dist}(c, X Y)
$$

ensures that the inverted " $\vee$ " $\langle b c d\rangle$ fits in $T_{\alpha}$ with $b$ and $d$ on opposite arms and $c$ on the base (Figure 10), and we adjust the notation as needed so that $c d \leq b c$. Angles $\angle c d Y$ and $\angle c b X$ are both obtuse, and a small counterclockwise rotation of $\langle b c d\rangle$ about $c$ moves $d$ to a point $d^{*}$ outside $T_{\alpha}$ and the point $b$ to a point $b^{*}$ inside $T_{\alpha}$. Since triangles $c b b^{*}$ and $c d d^{*}$ are similar and $c d \leq c b$, we see that $b b^{*} \geq d d^{*}$. Now reflect triangle $c b b^{*}$ through a line perpendicular to the base $X Y$ of $T_{\alpha}$, and translate the reflected image so that the image of $b$ is moved to the point $d$ (Figure 10), $b^{*}$ is moved to a point $r$, and $c$ to $c^{\prime}$. Let $s$ be the point so that $d$ is the midpoint of the segment $d^{*} s$. Then

$$
d r=b b^{*} \geq d d^{*}=d s .
$$

Let $d^{\prime}$ be the foot of the perpendicular from $d$ to $X Y$. Then the points $r$ and $s$ are clearly in the interior of $\angle d^{\prime} d Y$. Now

$$
\angle c^{\prime} d r=\angle c d d^{*}<90^{\circ}<\angle c d s \leq \angle c^{\prime} d s,
$$

and it follows that $r$ lies in the interior of $\angle d^{\prime} d s$. The point $r^{\prime}$ on the ray $d s$ at distance $b b^{*}$ from $d$ lies beyond $s$ on that ray. Temporarily write $\operatorname{xdist}(P, m)$ 


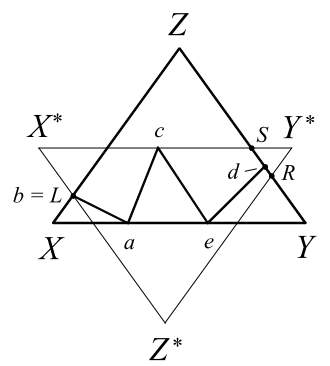

(a) $X^{*} Z^{*}$ touches $b$.

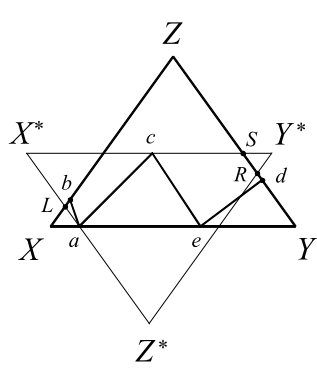

(b) $X^{*} Z^{*}$ touches $a$.

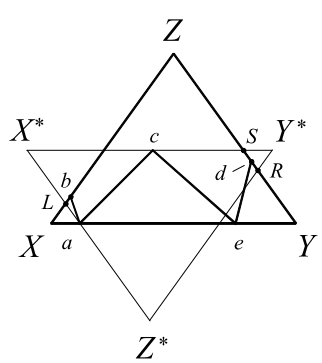

(c) $e$ beyond $Y^{*} Z^{*}$.

Figure 11: $w^{*}$-arcs.

for the horizontal distance from the point $P$ to the (non-horizontal) line $m$. Then

$$
\begin{aligned}
\operatorname{xdist}\left(b^{*}, X Z\right) & =x \operatorname{dist}(r, Y Z)>\operatorname{xdist}\left(r^{\prime}, Y Z\right) \geq \operatorname{xdist}(s, Y Z) \\
& =x \operatorname{dist}\left(d^{*}, Y Z\right) .
\end{aligned}
$$

Consequently, a small horizontal translation toward $X$ will move $\left\langle b^{*} c d^{*}\right\rangle$ so that both $b^{*}$ and $d^{*}$ are inside $T_{\alpha}$, and then a small vertical translation carries the inverted tetral $w$-arc $\pi$ into the interior of $T_{\alpha}$.

Finally we consider $w^{*}$-arcs, i.e., tetrals whose vertices are in the order $b \sim a \sim c \sim e \sim d$ and $\operatorname{dist}(b, X Y)+\operatorname{dist}(d, X Y)>\operatorname{dist}(c, X Y)$.

Lemma 13. No $w^{*}$-arc is an escape path.

Proof. Let $\pi=\langle$ baced $\rangle$ be a $w^{*}$-arc in $T_{\alpha}$, and with no loss of generality suppose

$$
\operatorname{dist}(b, X Y) \leq \operatorname{dist}(d, X Y) .
$$

Place an inverted copy $T_{\alpha}^{*}=X^{*} Y^{*} Z^{*}$ of $T_{\alpha}$ as pictured in Figure 11, with its base $X^{*} Y^{*}$ parallel to $X Y$ and with its arm $X^{*} Z^{*}$ touching the segment $b a$ on the left. Suppose that $X Z$ and $X^{*} Z^{*}$ meet at $L, X^{*} Y^{*}$ and $Y Z$ at $S$, and $Y Z$ and $Y^{*} Z^{*}$ at $R$. The point $e$ lies in the interior of $T_{\alpha}^{*}$ or it lies on or to the right of $Y^{*} Z^{*}$. In the former case the two possibilities, that $X^{*} Z^{*}$ touches $\pi$ at $b$ or at $a$, are illustrated in Figures 11(a) and (b). In (a),

$$
\operatorname{dist}(d, X Y)>\operatorname{dist}(c, X Y)-\operatorname{dist}(b, X Y)=\operatorname{dist}(R, X Y)
$$

so that $d$ lies on the segment $R S$ but not at $R$. Then a small translation clearly moves $\pi$ into the interior of $T_{\alpha}^{*}$. In (b), if $d$ lies on the segment $R S$ 
(but not at $R$ ), then $\pi=\langle$ baced $\rangle$ can be translated into the interior of $T_{\alpha}^{*}$, as in (a). Otherwise $d$ lies on the segment $R Y$,

$$
\operatorname{dist}(L, X Y)+\operatorname{dist}(d, X Y) \leq \operatorname{dist}\left(R, X^{*} Y^{*}\right)+\operatorname{dist}(R, X Y)=\operatorname{dist}(c, X Y),
$$

the arc $\langle$ Laced $\rangle$ is a $w$-arc, and $l(\langle$ baced $\rangle>l(\langle$ Laced $\rangle) \geq 1$ according to Lemma 11.

Suppose $e$ lies on or to the right of $Y^{*} Z^{*}$ (Figure 11(c)). If $d$ lies on the segment $R Y$ (but not at $R$ ), then irrespective of whether $X^{*} Z^{*}$ touches the segment $b a$ at $b$ or at $a$,

$$
l(\pi) \geq l(\langle\text { Laced }\rangle)>1
$$

If $d$ lies on the segment $S R$, then

$$
l(\pi) \geq l(\langle\text { Laced }\rangle) \geq l(\langle\text { Lace } R\rangle)>1 .
$$

This disposes of all the possibilities.

3.2.2. The family of all tetrals We consider the family of all tetrals. To simplify the notation, we write $x y$ to mean that the vertex $x$ precedes the vertex $y$ in the arclength parametrization of a tetral $\pi$. We lose no generality if we assume that $X \simeq a \sim e \simeq Y$ and ace. Now, there are 4 places for $b$, and, after $b$ is in place, 5 places for $d$, so there are 20 different tetral arcs to consider; and every tetral arc is essentially the same as one of those 20 (allowing always for two adjacent vertices to coincide where permitted). These 20 arcs fall into 7 groups of related arcs that yield to similar analyses. Reflecting a tetral arc $\pi$ through the altitude of $T_{\alpha}$ from $Z$ (and rearranging the labels as necessary) transforms $\pi$ into its reverse, and interchanging $b$ and $d$ in $\pi$ gives its swap, whose reverse is its inverse. For example, these arcs are illustrated in Figure 12 for the tetral arc $\pi=\langle$ bdace $\rangle$. For tetral arcs other than staples, $w$-arcs, and $w^{*}$-arcs these four arcs are generally different.

Table 1 gives the 20 tetral arcs assembled in related groups. An examination of the distinct tetral arcs in this table shows that all 20 possibilities are present, including both $w$-arcs and $w^{*}$-arcs.

Lemma 14. The only tetral arc in $T_{\alpha}$ that is an escape arc for $T_{\alpha}$ is $\mathfrak{z} \alpha$.

Proof. Note that the tetral arcs \#7 are staples and are longer then 1 by Lemma 9. Arcs \#3 are $s$-arcs and are fully described in Lemma 10; all have length at least 1 , and among them there is exactly one (up to congruence) 


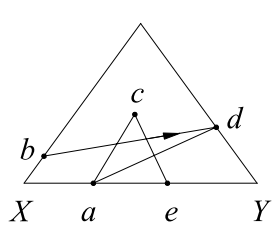

(a) bdace. Tetral arc $\pi$.

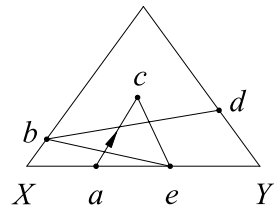

(b) acebd. Reverse of $\pi$.

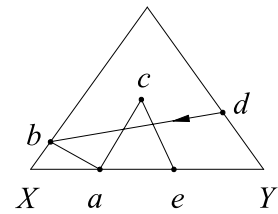

(c) dbace.

Swap of $\pi$.

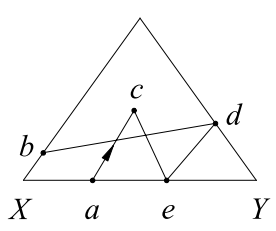

(d) acedb. Inverse of $\pi$.

Figure 12: The four related tetral arcs.

Table 1: The 7 essential tetral arcs and their related arcs

\begin{tabular}{|c|c|c|c|c|c|}
\hline & Tetral arc & Reverse & Swap & Inverse & \\
\hline 1. & bdace & acebd & dbace & $a c e d b$ & longer than a staple. \\
\hline 2. & badce & acbed & dabce & $a c d e b$ & longer than an $s$-arc. \\
\hline 3. & bacde & abced & dacbe & $a d c e b$ & $s$-arc. \\
\hline 4. & baced ${ }^{\dagger}$ & - & $d a c e b^{\dagger}$ & - & $w$-arc. \\
\hline 5. & baced $d^{\ddagger}$ & - & $d a c e b^{\ddagger}$ & - & $w^{*}$-arc. \\
\hline 6. & abdce & acbde & $a d b c e$ & acdbe & longer than a staple. \\
\hline 7. & abcde & - & adcbe & - & staple. \\
\hline
\end{tabular}

${ }^{\dagger}$ together with: $\operatorname{dist}(b, X Y)+\operatorname{dist}(d, X Y) \leq \operatorname{dist}(c, X Y)$.

$\ddagger$ together with: $\operatorname{dist}(b, X Y)+\operatorname{dist}(d, X Y)>\operatorname{dist}(c, X Y)$.

that is an escape arc, $\mathfrak{z} \alpha$. Arcs \#4 are $w$-arcs, and their behavior is fully described in Lemma 11; all have length at least one, and none is an escape arc. Arcs \#5 are $w^{*}$-arcs, and their behavior is described in Lemma 13. Only the groups of arcs listed in \#1, \#2, and \#6 remain to be examined.

Arcs \#1. Let $\pi=\langle$ bdace $\rangle$ (Figure 12). Let $b^{\prime}$ and $d^{\prime}$ be the feet of the perpendiculars from $b$ and $d$ to $X Y$, respectively. Then $b^{\prime} b \leq c a$ and $d^{\prime} d \leq c e$, so

$$
l(\pi)=b d+d a+a c+c e>b^{\prime} b+b d+d d^{\prime}=l\left(\left\langle b^{\prime} b d d^{\prime}\right\rangle>1,\right.
$$

because $\left\langle b^{\prime} b d d^{\prime}\right\rangle$ is a staple. The other arcs of the group are similar.

Arcs \#2. Let $\pi=\langle$ badce $\rangle$. Then

$$
\begin{aligned}
l(\pi) & =b a+a d+d c+c e \\
& >{ }_{1} b a+a c+c d+d e=l(\langle e d c a b\rangle) \geq_{2} 1 .
\end{aligned}
$$

The first inequality $\left(>_{1}\right)$ follows from $a d+c e>a c+d e$, an inequality that holds for every convex quadrilateral acde. The last inequality 
$\left(\geq_{2}\right)$ follows because $\langle$ bacde $\rangle$ (Arc \#3) is an $s$-arc. The other arcs of the group are similar.

Arcs \#6. Let $\pi=\langle a b d c e\rangle$. Define $b^{\prime}$ and $d^{\prime}$ as for Arcs \#1. Then $a b \geq b b^{\prime}$ and $d c+c e \geq d e \geq d d^{\prime}$, we see that

$$
l(\pi)=a b+b d+d c+c e \geq b^{\prime} b+b d+d d^{\prime}=l\left(\left\langle b^{\prime} b d d^{\prime}\right\rangle\right)>1
$$

because $\left\langle b^{\prime} b d d^{\prime}\right\rangle$ is a staple. The other arcs of the group are similar.

This completes the argument.

We have shown that there is exactly one tetral $\operatorname{arc}$ in $T_{\alpha}$ that is an escape path for $T_{\alpha}$, and it is $\mathfrak{z} \alpha$.

\section{The escape path}

Finally, we arrive at our principal result.

Theorem 15. The Besicovitch arc $\mathfrak{z} \alpha$ is the only escape path from a Besicovitch triangle $T_{\alpha}$.

Proof. The escape length $\varepsilon\left(T_{\alpha}\right)$ is 1 , and no exit path is shorter. Since the escape paths we seek lie in $T_{\alpha}$, they have unit length, and their endpoints are at least 0.59 apart, we lose no generality by restricting our attention to exit paths having these properties. Let $\gamma$ be such an exit path, with initial endpoint $P$ and terminal endpoint $Q$. According to Theorem $8, \gamma$ lies in an infinite strip $\sigma(m, n)$ of width $h, 0<h \leq \frac{1}{2}$, with two distinct points on $m$ and a third point between them on $n$. We picture the containing strip $\sigma(m, n)$ as horizontal, and we arrange for the initial point of $\gamma$ to lie to the left of the terminal point.

Place a triangle $T_{\alpha}=X Y Z$ with its base $X Y$ along $m$, its apex $Z$ on the same side of $m$ as $\gamma$, and its arm $X Z$ a support line of $\gamma$ (Figure 13). Then $\gamma$ lies in $\sigma(m, n) \cap \angle Y X Z$, and if $\gamma$ does not meet the arm $Y Z$ of $T_{\alpha}$, then a suitable small translation would move it into the interior of $T_{\alpha}$, a contradition.

Now, $\gamma$ has two distinct points $a$ and $e$ on the base $X Y$, a point $b$ on the $\operatorname{arm} X Z$, a point $d$ on the $\operatorname{arm} Y Z$, and a point $c$ on the line $n$ that lies between $a$ and $e$ in the parametric ordering of $\gamma$. Let $\pi_{\gamma}$ be the tetral arc that connects the five significant points $a, b, c, d, e$ in the same order as they lie on $\gamma$ (Figure 13). Then $\pi_{\gamma}$ is inscribed in $\gamma$, and

$$
1=l(\gamma) \geq l\left(\pi_{\gamma}\right) \geq 1
$$




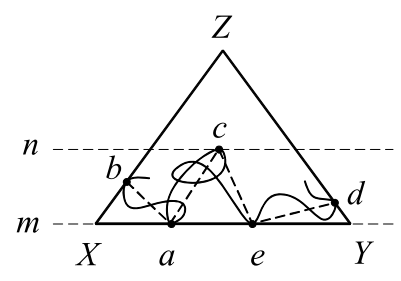

Figure 13: The argument.

since $\gamma$ has length 1 and $\pi_{\gamma}$ is inscribed in $\gamma$. Thus $\gamma$ must coincide with $\pi_{\gamma}$. But by Lemma 14, among the tetral arcs there is only one escape arc, viz., $\mathfrak{z} \alpha$.

In [12] one of us has extended the results presented here to isosceles triangles with base angle $\alpha$ in the range $45^{\circ} \leq \alpha<\alpha_{0}$, including the determination of the shortest base for which such a triangle is a cover for the family $\mathcal{C}_{1}$ of all unit arcs. In particular, this provides an affirmative answer to the conjecture in $[17$, p. 359] that an isosceles right triangle with base $\sqrt{10 / 9}$ is a cover for $\mathcal{C}_{1}$. It follows from arguments similar to those presented here that each such triangle has a unique escape path analogous to the Besicovitch path $\mathfrak{z} \alpha$.

Determining the escape paths for more general triangles seems to be quite a difficult geometric problem.

\section{References}

[1] Bellman, R. (1956). Minimization problem, Bull. Amer. Math. Soc. 62 270. MR1565769

[2] Besicovitch, S. (1965). On arcs that cannot be covered by an open equilateral triangle of side 1. Math. Gaz. 49 286. MR0188892

[3] Coulton, P. and Movshovich, Y. (2006). Besicovitch triangles cover unit arcs. Geom. Dedicata 123 79-88. MR2299727

[4] Dörrie, H. (1965). 100 Great Problems of Elementary Mathematics. Dover, New York.

[5] Eggleston, H. G. (1957). Problems in Euclidean Space: Applications of Convexity. Pergamon Press, London. MR0125931

[6] Finch, S. and Wetzel, J. E. (2004). Lost in a forest. Amer. Math. Monthly 111 645-654. MR2091541 
[7] Graham, R. (1963). Problem 41, in: Proc. 1963 Number Theory Conference, University of Colorado, Boulder, p. 98.

[8] Honsberger, R. (1970). Ingenuity in Mathematics. New Mathematical Library 23, Mathematical Association of America, Washington, DC.

[9] Maki, J. M., Wetzel, J. E. and Wichirimala, W. (2005). Drapeability. Discrete Comput. Geom. 34 637-657. MR2173931

[10] Moser, L. (1966). Poorly formulated unsolved problems in combinatorial geometry, mimeographed, undated, but about 1966.

[11] Moser, W. O. J. (1991). Problems, problems, problems. Discrete Appl. Math. 31 201-225. MR1106701

[12] Movshovich, Y. Besicovitch triangles extended (to appear in: Geom. Dedicata).

[13] Norwood, R., Poole, G. and Laidacker, M. (1992). The worm problem of Leo Moser. Discrete Comput. Geom. 7 153-162. MR1139077

[14] Poole, G. and Gerriets, J. (1973). Minimum covers for arcs of constant length. Bull. Amer. Math. Soc. 79 462-463. MR0315601

[15] Wetzel, J. E. (2010). An ancient elliptic locus. Amer. Math. Monthly 117 161-167. MR2590194

[16] Wetzel, J. E. (2005). The classical worm problem-a status report. Geombinatorics XV 34-42; Letter to the Editor, 92-93. MR2292763

[17] Wetzel, J. E. (2003). Fits and covers. Math. Mag. 76 349-363. MR2085394

[18] Wetzel, J. E. (1972). On Moser's problem of accommodating closed arcs in triangles. Elem. Math. 27 35-36. MR0295214

YEVGENYA MOVSHOVICH

Department of Mathematics and Computer Science

EASTERN ILLINOIS UNIVERSITY

600 Lincoln Avenue

Charleston, IL 61920-3099

USA

E-mail address: ymovshovich@eiu.edu 
JoHN E. WETZEL

Department of Mathematics

UNIVERSITY OF ILLINOIS AT URBANA-ChAMPAIGN

1409 W. Green Street

URBANA, IL 61801

USA

E-mail address: jewetz@comcast.net

Received September 27, 2010 